\title{
Aberrant expression of Notch1/numb/snail signaling, an epithelial mesenchymal transition related pathway, in adenomyosis
}

Shasha Qi ${ }^{1}$, Xingbo Zhao ${ }^{1}$, Mingjiang Li ${ }^{1}$, Xiaohui Zhang ${ }^{1}$, Zhenzhen Lu', Chunrun Yang ${ }^{1}$, Chunhua Zhang ${ }^{1}$, Hui Zhang ${ }^{1 *}$ and Na Zhang ${ }^{2}$

\begin{abstract}
Background: Epithelial mesenchymal transition (EMT) is involved in the pathogenesis of adenomyosis, and Notch signaling is crucial to EMT. The objective of this study was to explore Notch1/Numb/Snail signaling in adenomyosis.

Methods: The expression levels of the members of the Notch1/Numb/Snail signaling cascade in normal endometria (proliferative phase: $n=15$; secretory phase: $n=15$; postmenopausal phase: $n=15$ ) and adenomyotic endometria (proliferative phase: $n=15$; secretory phase: $n=15$ ) were determined by immunohistochemistry analysis.

Results: We found that the expressions of Notch1 and the EMT-related proteins N-cadherin, Snail and Slug were upregulated in the ectopic endometrium of adenomyosis compared with normal endometrium. Numb, a negative regulator of Notch signaling, was significantly decreased in adenomyosis. In addition, reduced immunoexpression of E-cadherin was observed in adenomyosis.
\end{abstract}

Conclusions: We conclude that Notch1/Numb/Snail signaling plays an important role in the pathogenesis and development of adenomyosis.

Keywords: Adenomyosis, Epithelial Mesenchymal Transition, Notch1/Numb/Snail Signaling, Slug

\section{Background}

Adenomyosis is a prevalent gynaecologic benign condition of the uterus characterized by the presence of activated endometrium within the myometrium [1]. The disease leads to dysmenorrhea, dyspareunia, abnormal uterine bleeding, and infertility and significantly reduces the quality of life of women of reproductive age [2]. The best treatment for adenomyosis is still unclear, and the mechanism of thisdisease has not been determined.

Epithelial-mesenchymal transition (EMT) is a biological process during which epithelial cells lose their polarity and cell-cell contacts and acquire a migratory mesenchymal phenotype [3, 4]. The process of EMT is characterized by the loss of epithelial markers and the acquisition of

\footnotetext{
*Correspondence: huizhang1218@126.com

'Department of Obstetrics and Gynecology, Shandong Provincial Hospital Affiliated to Shandong University, 324 Jingwu Road, Jinan, Shandong 250021, People's Republic of China

Full list of author information is available at the end of the article
}

mesenchymal markers [5]. The EMT plays a key role in tumour metastasis [6].

Migration and invasion are also considered key to the formation and progression of endometriosis [7]. Recent studies have shown that EMT-like processes may be involved in the pathogenesis of endometriosis $[8,9]$. Weaker expression of epithelial markers and stronger expression of mesenchymal markers are present in ectopic epithelial cells of endometriotic lesions on peritoneal and ovarian tissues [8]. In the epithelial component of adenomyotic lesions, vimentin expression is up-regulated and E-cadherin expression is down-regulated compared to the eutopic endometrium [10].

The Notch signaling pathway is thought to be critical for the induction of EMT. The Notch family, which included four members, Notch1-4, is a family of singlepass transmembrane receptor proteins [11]. Mature Notch receptors are heterologous dimers, consisting of a large extracellular ligand binding domain, a single-pass 
transmembrane structure and a small cytoplasmic subunit (Notch intracellular domain, NICD) [12, 13].Transmembrane ligands of the DSL (Delta/Serrate/Lag2) family bind to Notch receptors, triggering heterodimer cleavage and release of the NICD. The NICD then enters the nucleus and modulates the transcription of downstream target genes, including EMT-related genes, such as Snail and Slug (also called Snail2) [13]. Snail and Slug can combine with the E-cadherin promoter to suppress its expression $[14,15]$. Numb is an inhibitory regulator of Notch1 signaling that acts by promoting the ubiquitination and degradation of the Notch1 intracellular domain [16]. It has been reported that down-regulation or loss of Numb expression might be correlated with the genesis, development and enhancement of the invasion of multiple tumours $[17,18]$.

Notch signaling is involved in the process of EMT in a series of human tumours. Notch signaling can promote TGF- $\beta 1$-induced EMT through the induction of Snai1 [19]. In various human cancer models, Jagged1-mediated notch signaling activation can elevate the expressions of Snail and Slug, resulting in the repression of E-cadherin [20]. In pancreatic cancer cells, over-expression of Notch1 induces the EMT phenotype and increases cell growth, migration and invasion [21]. In lung cancer cells, inhibition of Notch signaling reverses the EMT process and, thus, enhances the therapeutic susceptibility of lung cancer cells [22]. In breast cancer cells, anti-human NICD monoclonal antibody can suppress the EMT process, inhibit cell growth and induce apoptosis [23]. Hypoxiainduced Notch signaling can affect EMT and migration of breast cancer cells by regulating the expression of Snail and Slug [24]. Moreover,Notch signaling can regulate the progression of metastatic hepatocellular carcinoma by regulating the expression of Snail and E-cadherin [25].

As shown above, Notch signaling cascades are crucial in the process of EMT. In the current study, we aimed to investigate the status of Notch $1 / \mathrm{Numb} /$ Snail signaling in adenomyosis and to explore the possible role of this signaling pathway in the development and progression of this disease.

\section{Methods}

\section{Materials and tissue collection}

Rabbit anti-human Notch1 (NICD); mouse anti-human Numb, E-cadherin, N-cadherin, and Slug; and goat antihuman Snail primary antibodies were obtained from Abcam (Beverly, MA, USA). Goat anti-rabbit and goat anti-mouse HRP-conjugated secondary antibodies and Diaminobenzidine staining kits were obtained from ZSGB-BIO (Beijing, China).

Normal endometria were obtained from 45 women of reproductive age undergoing bilateral tubal ligation (proliferative phase: $n=15$; secretory phase: $n=15$; postmenopausal phase: $n=15$ ). Adenomyotic lesions were obtained from 30 patients with adenomyosis undergoing hysterectomy or subtotal hysterectomy (proliferative phase: $n=15$; secretory phase: $n=15$ ) (Table 1). Normal endometria and adenomyosis tissues were collected during the operation.The diagnosis of adenomyosis was confirmed by histological examination. No patients received any hormonal therapy in three months prior to their surgery. Informed consent was obtained from all participants prior to the biopsy procedure, and the use of human tissues was approved by the institutional review board of Shandong Provincial Hospital Affiliated to Shandong University.

\section{Immunohistochemistry analysis}

Immunohistochemistry analysis was performed on normal endometria and adenomyotic lesions. Fresh tissue samples were washed with PBS twice to remove blood. Then, they were fixed in $4 \%$ paraformaldehyde for $24 \mathrm{~h}$ and embedded in paraffin. The samples were cut into $4 \mu \mathrm{m}$ sections and mounted onto glass slides. Deparaffinized,rehydrated sections were incubated with $3 \% \mathrm{H}_{2} \mathrm{O}_{2}$ for $30 \mathrm{~min}$ to block endogenous peroxidase activity. Antigen retrieval was performed using a pressure-cooker for 90 seconds inEDTA buffer at $\mathrm{pH}$ 7.6. The sections were rinsed in PBS, blocked with $10 \%$ normal goat serum or calf serum for $30 \mathrm{~min}$,and then incubated with primary antibodies, including rabbit anti-human notch(diluted 1:200 in PBS), mouse anti-human numb (diluted 1:150 in PBS), Ecadherin (diluted 1:100 in PBS), N-cadherin (diluted 1:500 in PBS), Slug (diluted 1:150 in PBS) andgoat anti-human snail (diluted 1:100 in PBS)antibodies, overnight in a wet chamber at $4{ }^{\circ} \mathrm{C}$. HRP-conjugated goat anti-rabbit or

Table 1 Detailed information of patients

\begin{tabular}{lllll}
\hline \multicolumn{2}{l}{ Proliferative phase } & & \multicolumn{2}{l}{ Secretory phase } \\
\cline { 5 - 5 } Ages & Dysmenorrhea & & Ages & Dysmenorrea \\
\hline 39 & + & 47 & + \\
44 & + & 41 & + \\
47 & + & 50 & + \\
44 & + & 45 & + \\
45 & + & 42 & + \\
43 & + & 42 & + \\
32 & + & 47 & + \\
47 & + & 37 & + \\
37 & + & 40 & + \\
43 & + & 48 & + \\
47 & + & 43 & + \\
44 & - & 52 & + \\
49 & + & 45 & + \\
39 & + & 48 & + \\
50 & + & 51 & + \\
\hline
\end{tabular}


mouse IgG was used as the second antibody, as appropriate. HRP activity was detected by measuring the level of the substrate diaminobenzidine tetrahydrochloride (DAB) for $1 \mathrm{~min}$. The sections were counterstained with haematoxylin before mounting. Sections incubated with nonimmune serum instead of primary antibodies were used as the negative controls. The sections were observed under a Leica DM4000B microscope (Leica), and pictures were taken using the IM50 image analysis system (Leica).

The immunostaining was expressed as immunoscore$\mathrm{H}$-score, which was semiquantitative as a product of a quantity score and a staining intensity. The quantity score was estimated as follows: four random views are chosen and 100 cells were counted to get the percentages $(\mathrm{Pi})$ of positively stained glandular epithelial cells. The staining intensity (I) of the glandular epithelial cells was estimated as follows: 0: negative; 1 : weak staining; 2 : moderate staining; and 3: strong staining. Two sections per sample were assessed by two observers. $\mathrm{H}$-score $=\mathrm{Pi}(\mathrm{I}+1)$. All slides were evaluated for immunostaining without any knowledge of the clinical or pathological data.

\section{Statistical analysis}

The data were statistically analysed by the two-tailed student's unpaired t-test using SPSS 19.0 (SPSS Inc., Chicago, IL). Values are expressed as means \pm SD. Differences between two groups were determined by the two-tailed student's t-test. The level of statistical significance was set at $p<0.05$.

\section{Results}

\section{Notch 1 expression was upregulated in adenomyosis}

The expression of Notch1 in normal endometria and ectopic endometria from adenomyotic lesions was determined using immunohistochemical analysis. As shown in Fig. 1, in normal endometria, the staining of Notch1 was weakly positive or positive and was concentrated in the cytoplasm of endometrial epithelial cells (Fig. 1, a-c). In stromal cells, the immunostaining of Notch1 was very weak. Endometria in the proliferative phase showed higher Notch1 expression than endometria in the secretory phase (Fig. 1a-b, $p<0.01$ ). No significant difference in Notch1 expression was noted between endometria in the productive phase and endometria in the postmenopausal phase (Fig. 1a-c, $p>0.05$ ).

In ectopic endometria of adenomyosis, the immunostaining of Notch1 was strongly positive and was also restricted to the cytoplasm of epithelial cells (Fig. 1d, e); weak immunostaining was observed in stromal cells. In addition, no significant difference in Notch1 expression was observed between ectopic endometria in the proliferative and secretory phases (Fig. 1d-e, $p>0.05$ ). However, ectopic endometria of adenomyosis in both the proliferative and secretory phases showed significantly increased Notch1 expression compared to normal endometria (Fig. 1f, $p<0.01$ ).

These data suggest that elevated Notch1 signaling is present in adenomyosis. Moreover, Notch1 expression was shown to change during the menstrual cycle in normal endometria but not in adenomyotic endometria.

\section{Numb expression was reduced in adenomyosis}

The expression of Numb in different endometria was determined by immunohistochemical analysis. As shown in Fig. 2, in normal endometria, the immunostaining of Numb was strongly positive and was most frequently distributed in the cytoplasm of endometrial epithelial cells; immunostaining in stromal cells was very weak. No significant difference in Numb expression was observed between endometria in the proliferative, secretory and postmenopausal phases (Fig. 2a-c, $p>0.05$ ).

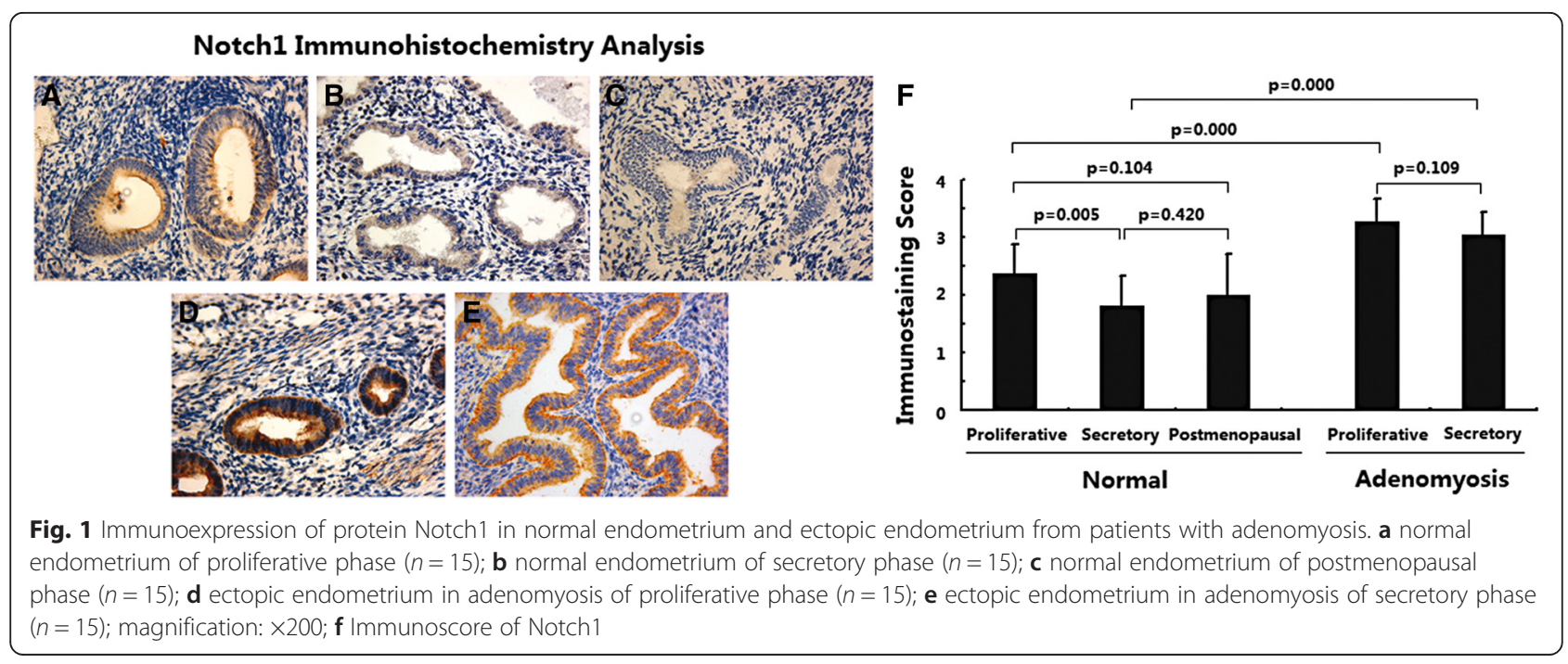




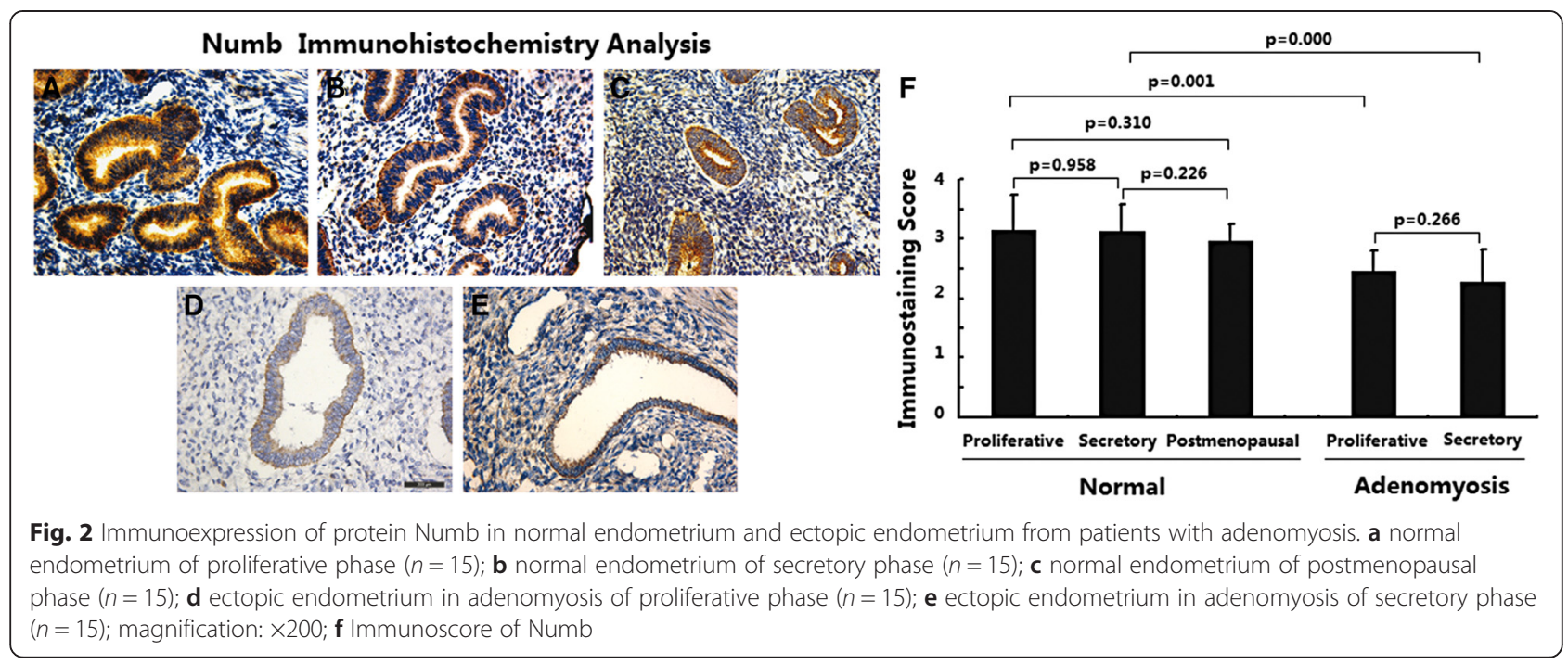

In ectopic endometria of adenomyosis, the immunostaining of Numb was weakly positive and was restricted to the cytoplasm of epithelial cells (Fig. 2d, e); weak immunostaining was observed in stromal cells. In addition, no significant difference in Numb expression was observed in ectopic endometria in the proliferative and secretory phases (Fig. 2d, e, $p>0.05$ ). However, ectopic endometria of adenomyosis showed significantly decreased Numb expression in both the proliferative and secretory phases compared with normal endometria (Fig. 2d, e, $p<$ 0.05).

These data suggest that Numb expression did not change during the menstrual cycles in either normal endometria or adenomyotic endometria and that Numb expression was lost in adenomyosis.
Snail expression was increased in adenomyosis

The expression of Snail in different endometria was determined using immunohistochemical analysis. As shown in Fig. 3, in normal endometria, the immunostaining of Snail was negative or weakly positive and was restricted to the nucleus of endometrial glandular epithelial cells; immunostaining in stromal cells was very weak. Endometria in the proliferative and postmenopausal phases showed decreased Snail expression compared with endometria in thesecretory phase (Fig. 3a-c, f, $p<0.01$ ). No significant difference in Snail expression was observed between endometria in the productive and postmenopausal phases (Fig. 3a-c, f, $p>0.05$ ).

In ectopic endometria of adenomyosis, the immunostaining of Snail was strongly positive and was restricted
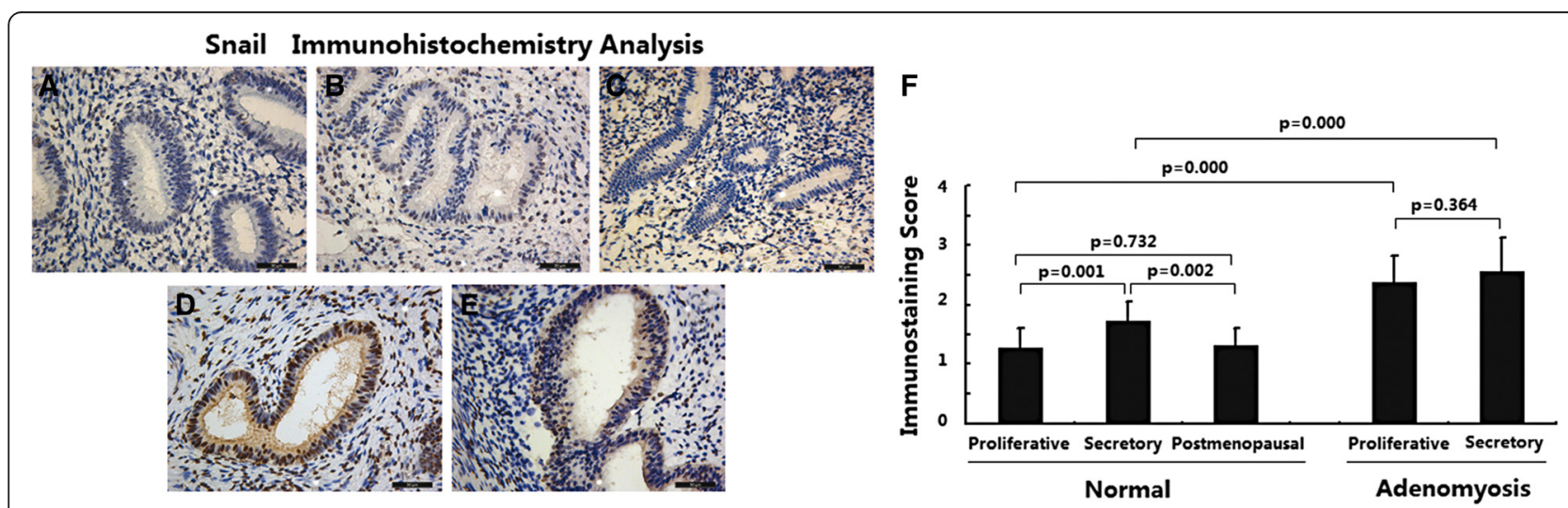

Fig. 3 Immunoexpression of protein Snail in normal endometrium and ectopic endometrium from patients with adenomyosis. a normal endometrium of proliferative phase $(n=15)$; $\mathbf{b}$ normal endometrium of secretory phase $(n=15)$; c normal endometrium of postmenopausal phase $(n=15)$; $\mathbf{d}$ ectopic endometrium in adenomyosis of proliferative phase $(n=15)$; e ectopic endometrium in adenomyosis of secretory phase $(n=15)$; magnification: $\times 200 ; \mathbf{f}$ Immunoscore of Snail 
to the nucleus of epithelial cells (Fig. 3d, e); weaker immunostaining was observed in stromal cells. No significant difference in Snail expression was observed between ectopic endometria in the proliferative and secretory phases (Fig. 3d-f, $p>0.05$ ); however, ectopic endometria of adenomyosis showed significantly increased Snail expression in both the proliferative and secretory phases compared with normal endometria(Fig. 3a-f, $p<0.01$ ).

These data suggest that Snail expression is elevated in adenomyosis. In addition, Snail expression changed during the menstrual cycle in normal endometriabut not in adenomyotic endometria.

\section{Slug expression was upregulated in adenomyosis}

The expression of Slug in different endometria was determined by immunohistochemical analysis. As shown in Fig. 4, in normal endometria, the immunostaining of Slug was weakly positive or positive and was usually distributed in the cell membrane of endometrial epithelial cells; immunostaining in stromal cells was very weak. No significant difference in Slug expression was observed between endometria in the proliferative, secretory and postmenopausal stages (Fig. 4a-c, $p>0.05$ ).

In ectopic endometria of adenomyosis, the immunostaining of Slug was also strongly positive and was restricted to the cell membrane of epithelial cells; weaker immunostaining was observed in stromal cells. No significant difference in Slug expression was observed between ectopic endometria in the proliferative and secretory phases (Fig. $4 \mathrm{~d}-\mathrm{f}, p>0.05$ ); however, ectopic endometria of adenomyosis in both the proliferative and secretory phases showed significantly increased Slug expression compared with normal endometria (Fig. 4d-f, $p<0.01$ ).

These data suggest that Slug expression did not change during the menstrual cycle in either normal endometria or adenomyotic endometria and that Slug expression was increased in adenomyosis.

\section{$\mathrm{N}$-cadherin expression was upregulated in adenomyosis}

The expression of $\mathrm{N}$-cadherin in different endometria was determined by immunohistochemical analysis. As shown in Fig. 5, in normal endometria, the immunostaining of $\mathrm{N}$-Cadherin was weakly positive or positive and was usually distributed in the membrane of endometrial epithelial cells; immunostaining in stromal cells was very weak. Endometria in the secretory and postmenopausal phases showed higher $\mathrm{N}$-cadherin expression than endometria in the proliferative phase (Fig. $5 \mathrm{a}-\mathrm{b}, p<0.05$ ). No significant difference in N-cadherin expression was observed between endometria in the secretory and postmenopausal phases (Fig. $5 \mathrm{a}-\mathrm{c}, p>0.05$ ).

In ectopic endometria of adenomyosis, the immunostaining of $\mathrm{N}$-cadherin was strongly positive and was restricted to the cytoplasm of epithelial cells (Fig. 5d, e); weak immunostaining was observed in stromal cells. No significant difference inN-cadherin expression was observed between ectopic endometria in the proliferative and secretory phases (Fig. $5 \mathrm{~d}-\mathrm{f}, p>0.05$ ); however, ectopic endometria of adenomyosis in both the proliferative and secretory phases showed significantly increased Ncadherin expression compared with normal endometria (Fig. 5f, $p<0.01$ ).

These data suggest that elevated $\mathrm{N}$-cadherin expression is present in adenomyosis. $\mathrm{N}$-cadherin expression changed during the menstrual cycles in normal endometria but not in adenomyotic endometria.

\section{E-cadherin expression was downregulated in adenomyosis}

The expression ofE-cadherinin different endometria was determined by immunohistochemical analysis. As shown

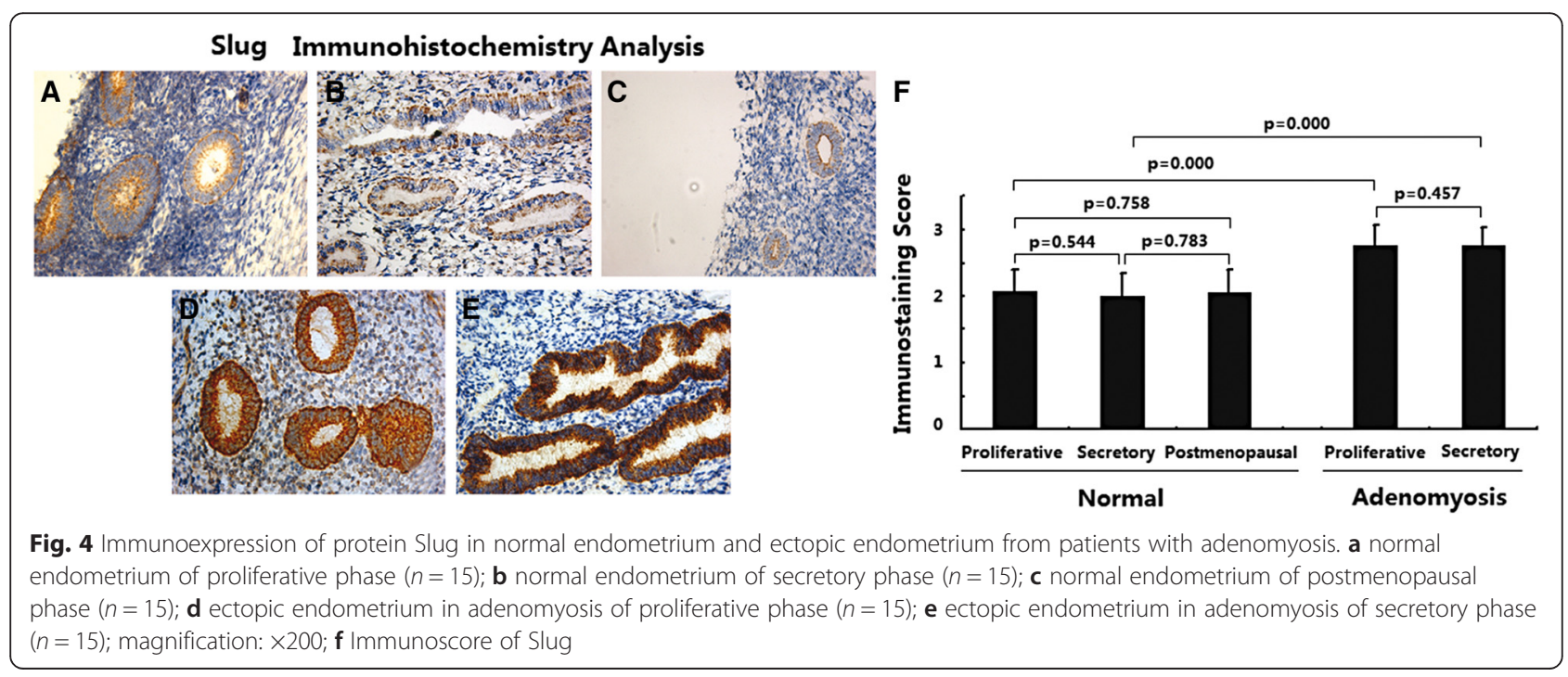




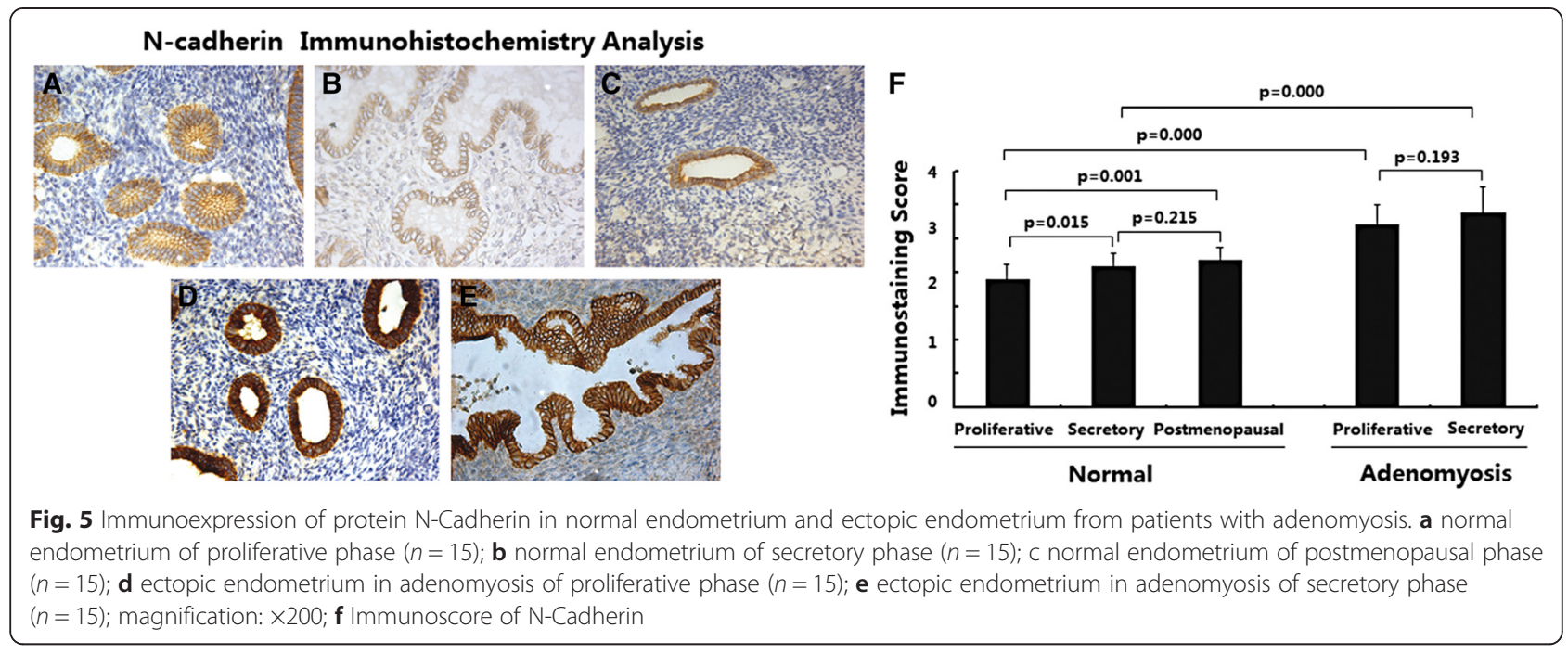

in Fig. 6, in normal endometria, the immunostaining of E-cadherin was strongly positive and was usually distributed in the membrane of endometrial epithelial cells; immunostaining in stromal cells was very weak. No significant difference of E-cadherin expression was observed between the endometria in different phases (Fig. 6a-c, f, $p>0.05$ ).

In ectopic endometria of adenomyosis, the immunostaining of E-cadherin was weakly positive and was restricted to the membrane of epithelial cells (Fig. 6d, e); weak immunostaining was observed in stromal cells. Ectopic endometria in the proliferative phase showed higher E-cadherin expression than ectopic endometria in the secretive phase (Fig. $6 \mathrm{~d}-\mathrm{f}, p<0.01$ ). In addition, ectopic endometria of adenomyosis showed significantly decreased E-cadherin expression in both the proliferative and secretory phases compared with normal endometria (Fig. 6d-f, $p<0.05$ ).
These data indicate that reduced E-cadherin expression is present in adenomyosis.

\section{Discussion}

Adenomyosis is adisease that exists independent from endometriosis [2]. The main pathological changes of adenomyosis are the invasion of functional endometrial glands and stroma into the myometrium and the growth of ectopic glands or stroma in the myometrium and/or local hyperplasia [1]. Although adenomyosis is a benign disease, it exhibits a series of biological behaviours that are similar to those of malignant tumours, including adhesion, invasion, and implantation [26]. EMT is a process during which epithelial cells undergo phenotypic transformation into mesenchymal cells [5]. A great dealof evidence indicates that EMT is associated with the invasive and migratory behaviours of cancer cells, which enhance the metastatic ability of these cells [6, 7]. Chen et al.
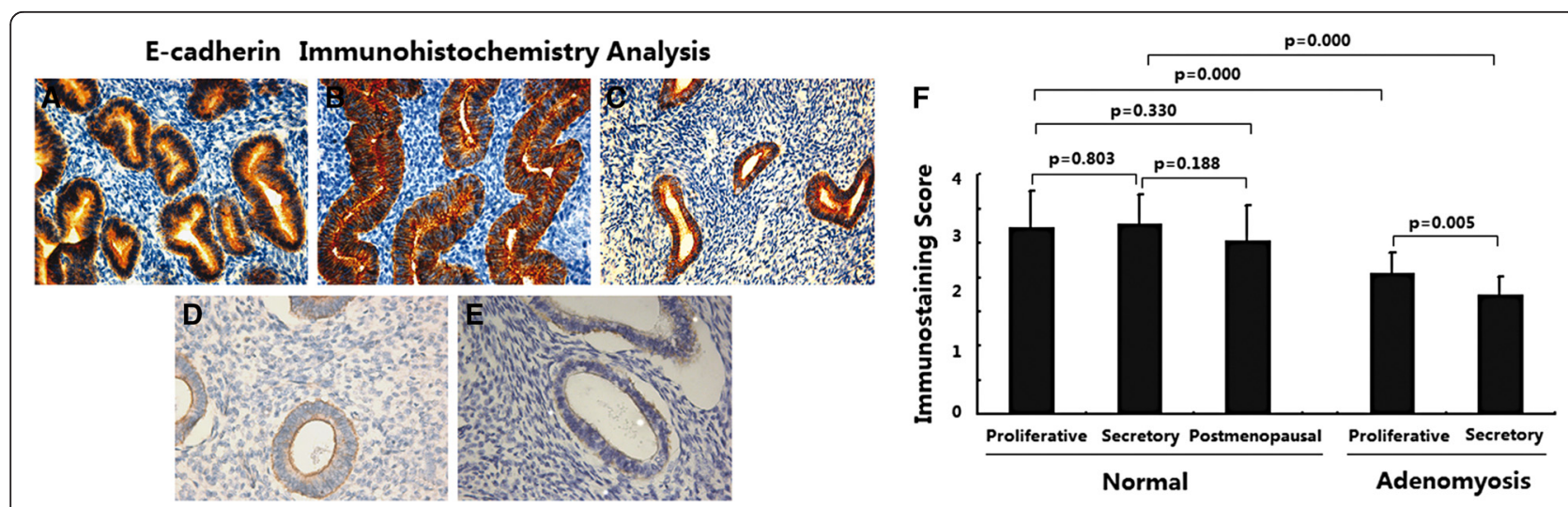

Fig. 6 Immunoexpression of protein E-Cadherin in normal endometrium and ectopic endometrium from patients with adenomyosis. a normal endometrium of proliferative phase $(n=15)$; $\mathbf{b}$ normal endometrium of secretory phase $(n=15)$; c normal endometrium of postmenopausal phase $(n=15)$; $\mathbf{d}$ ectopic endometrium in adenomyosis of proliferative phase $(n=15)$; e ectopic endometrium in adenomyosis of secretory phase $(n=15)$; magnification: $\times 200 ; \mathbf{f}$ Immunoscore of E-Cadherin 


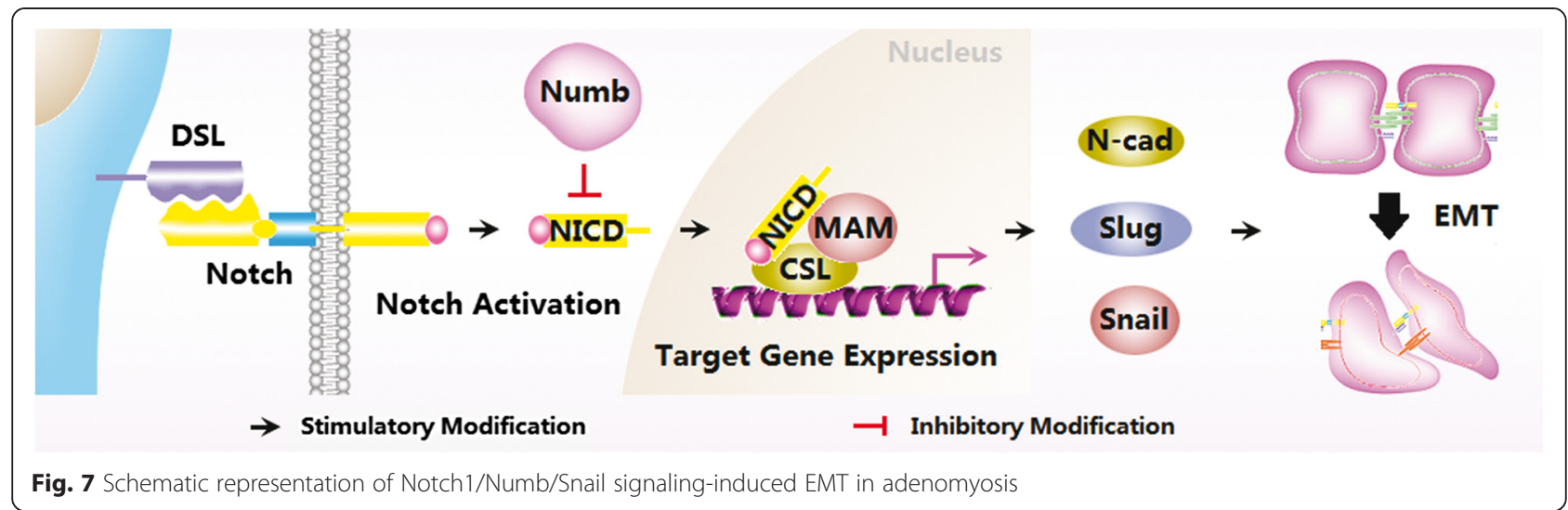

reported that EMT markers are aberrantly expressed in adenomyosis [10]. In the current study, we found that the EMT-related Notch1/Numb/Snail signaling pathway plays an important role in the pathogenesis of adenomyosis.

Notch signalingis involved in cell proliferation, survival, apoptosis, and differentiation, and alterations in Notch signaling are linked to tumourigenesis [27]. Notch activation in endothelial cells results in the down-regulation of endothelial markers and the up-regulation of mesenchymal markers [28]. In the EMT process, Notch signalingcrosstalks with multiple transcription and growth factors that are relevant to EMT, such as Snail, Slug, TGF- $\beta$, FGF, and PDGF $[29,30]$.

In human endometrium, Notch1-3 are expressed not only stromal cells but also in glandular epithelial cells, and Jagged and DDL4 are mainly expressed in glandular epithelial cells [31]. Mori et al. reported that the expression of Notch1 in the endometrium is higher during the proliferative phase than the secretory phase and is lowest during the postmenopausal phase [32]. In contrast, Cobellis et al. found that the expressions of Notch1 and Jagged1 increased from the proliferative phase to the secretory phase [33]. Notch1 plays an important role in the differentiation and decidualization of endometrial stromal cells [34]. During this process, the expression of Notch1 is downregulated and the expression of Numb is up-regulated [35]. In endometrial carcinoma, the expressions of Notch, Jagged1, and DLL4 are significantly increased and are related to the stage and prognosis of the disease, and blockage of the Notch signaling pathway significantly inhibits the growth and invasion of endometrial adenocarcinoma cells [32, 36]. In addition, blockage of Notch signaling induces apoptosis in Ishikawa cells [37], while increased oestrogen promotes the growth of Ishikawa cells by activating the Notch signaling pathway [38]. In the current study, elevated Notch1 expression was noted in adenomyosis, suggesting its significant role in this disease. We found that endometrium in the proliferative phase showed higher Notch1 expression than that in the secretory phase and that endometrium in the postmenopausal phase showed the lowest level of Notch1 expression. These data are consistent with those of the study by Mori et al. Moreover, in our study, Notch1 expression changed during the menstrual cycle in normal endometria but not in adenomyotic endometria. These data indicate that of Notch1 expression in normal endometrium is regulated by hormones and that this hormonal sensitivity is aberrant in adenomyosis.

Numb protein was first observed in Drosophila [39] and is thought to be a cell fate determinant that acts by regulating cell division, adhesion, and migration [40] In mammals, Numb protein inhibits Notch signaling by promoting the ubiquitination of the Notch1 receptor and the degradation of Notch1 intracellular domain (NICD) [16]. Numb is considered a tumour suppressor [41] in various carcinomas, including breast cancer [42] and salivary gland carcinomas [43]. However,Numb overexpression has been observed in astrocytomas [44] and cervical squamous carcinoma cells [45], implying that Numb may be oncogene in these diseases. In the current study, we investigated Numb expression in normal endometrium and adenomyotic endometrium, and we found that Numb expression did not change during the menstrual cycle in either normal endometria or adenomyotic endometria, indicating that Numb expression is hormonally independent. In addition, the loss of Numb expression was noted in adenomyosis, demonstrating that aberrant negative regulation of Numb may be involved in the genesis and development of adenomyosis. To our knowledge, the current study is the first to explore the role of Numb in adenomyosis.

EMT is activated by a number of transcription factors, including Snail, Slug, and Twist, and also by the repression of E-cadherin expression [46]. Snail and Slug have been reported to be associated with tumour cell migration, invasion, and metastasis. Snail was first discovered in Drosophila as a zinc-finger transcription factor and has since been proven to be a key regulator of EMT [47]. Snail 
also represses E-cadherin transcription by binding to the E-box site in the promoter of E-cadherin [48]. The role of Snail in EMT regulation has been reported in multiple carcinoma types, including breast carcinoma, ovarian carcinoma, etc. [48, 49]. Slug, which belongs to the Slug family of zing-finger transcription factors, also plays a major role in EMT during embryonic development and metastasis of various cancers by inhibiting E-cadherin [50]. In ovarian carcinoma cells, increased expression of Snail and Slug directly lead to cisplatin resistance [51] and promote the EMT process by activating the $\beta$-Catenin-T-Cell Factor-4dependent expression of transforming growth factor- $\beta 3$ [52]. Functional knockdown of Snail and Slug was shown to significantly decrease the tumourigenicity and metastatic behaviour of squamous carcinoma cells [53]. In the current study, we discovered that Snail and Slug were upregulated in adenomyosis, indicating the possible role of Snail/Slugassociated EMT in the pathogenesis and development of adenomyosis. In addition, Snail expression changed during the menstrual cycle in normal endometria, but Slug expression did not change during the menstrual cycle in either normal endometria or adenomyotic endometria. Furthermore, the menstrual changes in Snail expression were absent in adenomyosis, suggesting the decreased hormonal sensitivity of the ectopic endometrium of adenomyosis.

$\mathrm{N}$-cadherin is another EMT marker. A switch from expression of E-cadherin to expression of $\mathrm{N}$-cadherin is frequently observed in many aggressive cancers [27]. Ncadherin stimulates the upregulation of Snail and Slug in a FGFR-dependent manner [54]. N-cadherin-mediated cell adhesion accelerates cell migration in a three-dimensional matrix [55]. In adenomyosis, we found that $\mathrm{N}$-cadherin was up-regulated in ectopic epithelial cells, indicating the important role of $\mathrm{N}$-cadherin in this disease. Moreover, $\mathrm{N}$ cadherin expression changed during the menstrual cycle in normal endometrium but not in adenomyotic endometrium. These data suggest decreased hormonal sensitivity in adenomyosis.

One of the most common features of EMT is the loss of E-cadherin expression [27]. During the EMT process, epithelial cells undergo a phenotypic switch to the mesenchymal phenotype, which leads to the loss of cell-cell adhesion, alternation of polarity, modulation of the cytoskeletal systems, and a switch of expression from keratin to vimentin [5]. Inhibition of Snail may stimulate the re-expression of E-cadherin and other epithelial markers in metastatic tissues, where higher expression of E-cadherin and epithelial characteristics may contribute to increased survival and proliferation [56]. In prostate cancer, E-cadherin and Snail levels can be measured to assess disease prognosis and can be used as therapeutic targets to prevent metastatic progression [57].A previous study reported that E-cadherin expression was decreased in the uterus of mice and in human adenomyotic lesions [56]. Consistent with the results of Shih et al., in the current study, the expression of Ecadherin was significantly reduced in ectopic epithelial cells of adenomyotic endometrium. In addition, E-cadherin expression showed no hormonal dependence in normal endometrium, while higher E-cadherin expression was noted in adenomyotic endometrium in the proliferative phase compared than in adenomyotic endometrium in the secretory phase.

\section{Conclusion}

In conclusion, our data demonstrate the possible involvement of Notch1/Snail/Numb signaling in the pathogenesis and development of adenomyosis (Fig. 7). The current study may provide new insight into the diagnosis and treatment of adenomyosis. However, the main limitation of this study is that we only examined the expression and location of Notch1/Numb/Snail signaling by immunohistochemistry. In our next study, our team will examine the involvement of Notch1 signaling in adenomyosis using multiple experimental techniques.

\section{Competing interests}

The authors declare that they have no competing interests.

\section{Authors' contributions}

$\mathrm{HZ}$ conceived of the study, participated in its design and coordination, helped to draft the manuscript and edit the manuscript for submission. SQ carried out the immunoassays, participated in the analysis and the interpretation of data and drafted the manuscript. XZ participated in the design of the study, supervised the study and critically helped to draft the manuscript. ML contributed to the design of the study, assisted in data analysis and revised the manuscript. NZ helped to revise the manuscript. $X Z, Z L, C Y$ and $C Z$ performed the statistical analysis. All authors read and approved the final manuscript.

\section{Acknowledgements}

The research was supported by grants from the National Natural Science Foundation of China (No.81300468; No.81272858;No.81170549) and grants from Shandong Province excellent youth scientist foundation

(No.BS2013YY008; No.2009BSB14147). We would also like to acknowledge the professional manuscript services of American Journal Experts.

\section{Author details}

'Department of Obstetrics and Gynecology, Shandong Provincial Hospital Affiliated to Shandong University, 324 Jingwu Road, Jinan, Shandong 250021, People's Republic of China. ${ }^{2}$ Department of Anesthesiology and Surgery, Shandong Provincial Hospital Affiliated to Shandong University, 324 Jingwu Road, Jinan, Shandong 250021, People's Republic of China.

Received: 7 April 2015 Accepted: 28 July 2015

Published online: 26 August 2015

\section{References}

1. Guo Q, Zhang H, Zhao X, Fu Y, Zhang J, Li M. Loss of expressions of Dusp6, Sprouty4, and Sef, negative regulators of FGF2/ERK1/2 signaling, in the endometrium of women with adenomyosis. Int J Gynecol Pathol. 2014;33:288-97.

2. Di Donato N, Seracchioli R. How to evaluate adenomyosis in patients affected by endometriosis? Minim Invasive Surg. 2014;2014:507230.

3. Marie-Egyptienne DT, Lohse I, Hill RP. Cancer stem cells, the epithelial to mesenchymal transition (EMT) and radioresistance: potential role of hypoxia. Cancer Lett. 2013;341:63-72.

4. Voulgari A, Pintzas A. Epithelial-mesenchymal transition in cancer metastasis: mechanisms, markers and strategies to overcome drug resistance in the clinic. Biochim Biophys Acta. 2009;1796:75-90. 
5. Iwatsuki M, Mimori K, Yokobori T, Ishi H, Beppu T, Nakamori S, et al. Epithelial-mesenchymal transition in cancer development and its clinical significance. Cancer Sci. 2010;101:293-9.

6. Son H, Moon A. Epithelial-mesenchymal transition and cell invasion. Toxicol Res. 2010;26:245-52.

7. Giudice LC, Kao LC. Endometriosis. Lancet. 2004;364:1789-99.

8. Matsuzaki S, Darcha C. Epithelial to mesenchymal transition-like and mesenchymal to epithelial transition-like processes might be involved in the pathogenesis of pelvic endometriosis. Hum Reprod. 2012;27:712-21.

9. Santamaria X, Massasa EE, Taylor HS. Migration of cells from experimental endometriosis to the uterine endometrium. Endocrinology. 2012;153:5566-74.

10. Chen YJ, Li HY, Huang CH, Twu NF, Yen MS, Wang PH, et al. Oestrogeninduced epithelial-mesenchymal transition of endometrial epithelial cells contributes to the development of adenomyosis. J Pathol. 2010;222:261-70.

11. Wang Z, Li Y, Kong D, Sarkar FH. The role of notch signaling pathway in Epithelial-Mesenchymal Transition (EMT) during development and tumor aggressiveness. Curr Drug Targets. 2010;11:745-51.

12. Groot AJ, Vooijs MA. The role of Adams in notch signaling. Adv Exp Med Biol. 2012;727:15-36.

13. Chan YM, Jan YN. Roles for proteolysis and trafficking in Notch maturation and signal transduction. Cell. 1998;94:423-6.

14. Cano A, Perez-Moreno MA, Rodrigo I, Locascio A, Blanco MJ, Del Barrio MG, et al. The transcription factor snail controls epithelial-mesenchymal transitions by repressing E-cadherin expression. Nat Cell Biol. 2000;2:76-83.

15. Bolos V, Peinado H, Perez-Moreno MA, Fraga MF, Esteller M, Cano A. The transcription factor Slug represses E-cadherin expression and induces epithelial to mesenchymal transitions: a comparison with Snail and E47 repressors. J Cell Sci. 2003;116:499-511.

16. Mcgill MA, Mcglade CJ. Mammalian numb proteins promote Notch1 receptor ubiquitination and degradation of the Notch1 intracellular domain. J Biol Chem. 2003:278:23196-203.

17. Jiang $X$, Xing $H$, Kim TM, Jung $Y$, Huang $W$, Yang HW, et al. Numb regulates glioma stem cell fate and growth by altering epidermal growth factor receptor and Skp1-Cullin-F-box ubiquitin ligase activity. Stem Cells. 2012;30:1313-26.

18. Ito $\mathrm{T}$, Kwon HY, Zimdahl B, Congdon KL, Blum J, Lento WE, et al. Regulation of myeloid leukaemia by the cell-fate determinant Musashi. Nature. 2010;466:765-8

19. Matsuno Y, Coelho AL, Jarai G, Westwick J, Hogaboam CM. Notch signaling mediates TGF-beta1-induced epithelial-mesenchymal transition through the induction of Snai1. Int J Biochem Cell Biol. 2012:44:776-89.

20. Leong KG, Niessen K, Kulic I, Raouf A, Eaves C, Pollet I, et al. Jagged1mediated Notch activation induces epithelial-to-mesenchymal transition through Slug-induced repression of E-cadherin. J Exp Med. 2007;204:2935-48

21. Bao B, Wang Z, Ali S, Kong D, Li Y, Ahmad A, et al. Notch-1 induces epithelial-mesenchymal transition consistent with cancer stem cell phenotype in pancreatic cancer cells. Cancer Lett. 2011:307:26-36.

22. Xie M, Zhang L, He CS, Xu F, Liu JL, Hu ZH. Activation of Notch-1 enhances epithelial-mesenchymal transition in gefitinib-acquired resistant lung cancer cells. J Cell Biochem. 2012;113:1501-13.

23. Sharma A, Paranjape AN, Rangarajan A, Dighe RR. A monoclonal antibody against human Notch1 ligand-binding domain depletes subpopulation of putative breast cancer stem-like cells. Mol Cancer Ther. 2012;11:77-86

24. Chen J, Imanaka N, Chen J, Griffin JD. Hypoxia potentiates Notch signaling in breast cancer leading to decreased E-cadherin expression and increased cell migration and invasion. Br J Cancer. 2010;102:351-60.

25. Wang $X Q$, Zhang W, Lui EL, Zhu Y, Lu P, Yu X, et al. Notch1-Snail1-Ecadherin pathway in metastatic hepatocellular carcinoma. Int J Cancer. 2012;131:E163-72

26. Leyendecker $G$, Wildt $L$, Mall G. The pathophysiology of endometriosis and adenomyosis: tissue injury and repair. Arch Gynecol Obstet. 2009;280:529-38

27. Miele L. Notch signaling. Clin Cancer Res. 2006;12:1074-9.

28. Chang AC, Garside VC, Fournier M, Smrz J, Vrljicak P, Umlandt P, et al. A Notch-dependent transcriptional hierarchy promotes mesenchymal transdifferentiation in the cardiac cushion. Dev Dyn. 2014;243:894-905.

29. Gonzalez DM, Medici D. Signaling mechanisms of the epithelial-mesenchymal transition. Sci Signal. 2014;7:re8.
30. Wu K, Chen K, Wang C, Jiao X, Wang L, Zhou J, et al. Cell fate factor DACH1 represses YB-1-mediated oncogenic transcription and translation. Cancer Res. 2014;74:829-39.

31. Mikhailik A, Mazella J, Liang S, Tseng L. Notch ligand-dependent gene expression in human endometrial stromal cells. Biochem Biophys Res Commun. 2009;388:479-82.

32. Mori M, Miyamoto T, Ohno S, Miyake Y, Sakaguchi T, Ohno E. Diagnostic utility of notch-1 immunocytochemistry in endometrial cytology. Acta Cytol. 2012;56:166-70.

33. Cobellis L, Caprio F, Trabucco E, Mastrogiacomo A, Coppola G, Manente L et al. The pattern of expression of Notch protein members in normal and pathological endometrium. J Anat. 2008;213:464-72.

34. Afshar Y, Jeong JW, Roqueiro D, Demayo F, Lydon J, Radtke F, et al. Notch1 mediates uterine stromal differentiation and is critical for complete decidualization in the mouse. FASEB J. 2012:26:282-94.

35. Afshar Y, Miele L, Fazleabas AT. Notch1 is regulated by chorionic gonadotropin and progesterone in endometrial stromal cells and modulates decidualization in primates. Endocrinology. 2012;153:2884-96.

36. Mitsuhashi Y, Horiuchi A, Miyamoto T, Kashima H, Suzuki A, Shiozawa T. Prognostic significance of Notch signalling molecules and their involvement in the invasiveness of endometrial carcinoma cells. Histopathology. 2012;60:826-37.

37. Mori M, Miyamoto T, Yakushiji H, Ohno S, Miyake Y, Sakaguchi T, et al. Effects of $\mathrm{N}$-[N-(3, 5-difluorophenacetyl-L-alanyl)]-S-phenylglycine t-butyl ester (DAPT) on cell proliferation and apoptosis in Ishikawa endometrial cancer cells. Hum Cell. 2012;25:9-15.

38. Wei Y, Zhang Z, Liao H, Wu L, Wu X, Zhou D, et al. Nuclear estrogen receptor-mediated Notch signaling and GPR30-mediated PI3K/AKT signaling in the regulation of endometrial cancer cell proliferation. Oncol Rep. 2012:27:504-10

39. Uemura T, Shepherd S, Ackerman L, Jan LY, Jan YN. Numb, a gene required in determination of cell fate during sensory organ formation in Drosophila embryos. Cell. 1989;58:349-60.

40. Rasin MR, Gazula VR, Breunig JJ, Kwan KY, Johnson MB, Liu-Chen S, et al. Numb and Numbl are required for maintenance of cadherin-based adhesion and polarity of neural progenitors. Nat Neurosci. 2007;10:819-27.

41. Mcgill MA, Dho SE, Weinmaster G, Mcglade CJ. Numb regulates postendocytic trafficking and degradation of Notch1. J Biol Chem. 2009;284:26427-38

42. Rennstam K, Mcmichael N, Berglund P, Honeth G, Hegardt C, Ryden L, et al. Numb protein expression correlates with a basal-like phenotype and cancer stem cell markers in primary breast cancer. Breast Cancer Res Treat. 2010;122:315-24

43. Maiorano E, Favia G, Pece S, Resta L, Maisonneuve P, Di Fiore PP, et al. Prognostic implications of NUMB immunoreactivity in salivary gland carcinomas. Int J Immunopathol Pharmacol. 2007;20:779-89.

44. Yan B, Omar FM, Das K, Ng WH, Lim C, Shiuan K, et al. Characterization of Numb expression in astrocytomas. Neuropathology. 2008;28:479-84.

45. Chen $\mathrm{H}$, Chen X, Ye F, Lu W, Xie X. Symmetric division and expression of its regulatory gene Numb in human cervical squamous carcinoma cells. Pathobiology. 2009:76:149-54

46. Thiery JP. Epithelial-mesenchymal transitions in tumour progression. Nat Rev Cancer. 2002;2:442-54.

47. Nieto MA. The snail superfamily of zinc-finger transcription factors. Nat Rev Mol Cell Biol. 2002;3:155-66.

48. Chenfang Dong YW. Jun Yao, Yifan Wang, Yinhua Yu, Piotr G. Rychahou, B. Mark Evers, Binhua P. Zhou: G9a interacts with Snail and is critical for Snail-mediated E-cadherin repression in human breast cancer. J Clin Invest. 2012;122(4):1469-86

49. Pon YL, Zhou HY, Cheung AN, Ngan HY, Wong AS. p70 S6 kinase promotes epithelial to mesenchymal transition through snail induction in ovarian cancer cells. Cancer Res. 2008:68:6524-32.

50. Kudo-Saito C, Shirako H, Takeuchi T, Kawakami Y. Cancer metastasis is accelerated through immunosuppression during Snail-induced EMT of cancer cells. Cancer Cell. 2009;15:195-206.

51. Haslehurst AM, Koti M, Dharsee M, Nuin P, Evans K, Geraci J, et al. EMT transcription factors snail and slug directly contribute to cisplatin resistance in ovarian cancer. BMC Cancer. 2012:12:91.

52. Medici D, Hay ED, Olsen BR. Snail and Slug promote epithelial-mesenchymal transition through beta-catenin-T-cell factor-4-dependent expression of transforming growth factor-beta3. Mol Biol Cell. 2008;19:4875-87. 
53. Olmeda D, Montes A, Moreno-Bueno G, Flores JM, Portillo F, Cano A. Snai1 and Snai2 collaborate on tumor growth and metastasis properties of mouse skin carcinoma cell lines. Oncogene. 2008;27:4690-701.

54. Qian X, Anzovino A, Kim S, Suyama K, Yao J, Hulit J, et al. N-cadherin/FGFR promotes metastasis through epithelial-to-mesenchymal transition and stem/progenitor cell-like properties. Oncogene. 2014:33:3411-21.

55. Shih W, Yamada S. N-cadherin-mediated cell-cell adhesion promotes cell migration in a three-dimensional matrix. J Cell Sci.

2012;125:3661-70.

56. Putzke AP, Ventura AP, Bailey AM, Akture C, Opoku-Ansah J, Celiktas M, et al. Metastatic progression of prostate cancer and e-cadherin regulation by zeb1 and SRC family kinases. Am J Pathol. 2011;179:400-10.

57. Deep G, Jain AK, Ramteke A, Ting H, Vijendra KC, Gangar SC, et al. SNAl1 is critical for the aggressiveness of prostate cancer cells with low E-cadherin. Mol Cancer. 2014;13:37.

\section{Submit your next manuscript to BioMed Central and take full advantage of:}

- Convenient online submission

- Thorough peer review

- No space constraints or color figure charges

- Immediate publication on acceptance

- Inclusion in PubMed, CAS, Scopus and Google Scholar

- Research which is freely available for redistribution 